\title{
Relaxation Time and Effective Mass of Ions in Liquid Helium*
}

\author{
A. J. Dahm $\dagger$ \\ School of Physics, University of Minnesota, Minneapolis, Minnesota \\ and \\ T. M. Sanders, Jr. \\ H. M. Randall Laboratory, University of Michigan, Ann Arbor, Michigan
}

(Received November 5, 1969)

\begin{abstract}
A measurement of momentum relaxation times for charged particles in liquid helium is reported. The method is to measure the change in the reflection coefficient of a microwave cavity caused by the admission of ions. The measured relaxation times can be combined with the known dc mobilities of the ions to yield values for the effective masses. Most extensive measurements were performed using positive ions in $\mathrm{He}$ II. A temperature-dependent effective mass, increasing from approximately 40 helium masses at $1.3 \mathrm{~K}$ to over twice this value near the lambda point is found. The data are shown to be consistent with the electrostriction model of the ion, including contributions to the mass from both normal and superfluid components. $A$ core radius of approximately $6 \AA$ is shown to account both for the mass and for the mobility in the viscous flow region. Data on the negative carrier near $1.8 \mathrm{~K}$ yield an effective mass of 100 to 200 helium masses, and are consistent with the bubble model. The radius implied boi h by the mass and an analysis of mobility data in the viscous flow regime is $14 \pm 3 A$, quite consistent with the value deduced from other measurements. A measurement on the positive carrier in saturated (NBP) helium vapor indicates a mass of $75 \pm 20$ helium masses. Both the mass and the mobility of this carrier are consistent with a droplet of radius $11 \pm 2 \AA$ forming on the ion. The theoretical droplet radius is $12.6 \AA$.
\end{abstract}

\section{INTRODUCTION}

In the experiments that have been performed using charged particles in liquid helium, considerably more emphasis has been placed on what one can learn about the liquid than on the structure of the charged particles themselves. Over the years, a number of experiments bearing on the nature of the carriers have been reported; nonetheless many unsettled questions have lingered on, and a variety of different models of the ions have received theoretical attention. Prominent among the measurable structure-sensitive quantities is the effective mass.

\footnotetext{
*This work was supported in part by the Air Force Office of Scientific Research, The U.S. Atomic Energy Commission, and Research Corporation.

$\dagger$ Present address: Department of Physics, Case Western Reserve University, Cleveland, Ohio.
} 
This quantity might be obtained directly by the cyclotron resonance technique, a possibility that several groups have explored, but to date this method has not yielded any results.

We decided to make a somewhat indirect evaluation of the effective masses, by measuring momentum relaxation times. ${ }^{1}$ Such quantities can be combined with the known mobilities to yield values for the carrier masses. This type of measurement appeared to offer some advantages over the cyclotron resonance experiment, in spite of the fact that it cannot determine the masses as accurately; the measurements could be carried out in the temperature range accessible by pumping on liquid ${ }^{4} \mathrm{He}$, using apparatus we already had on hand. It was our initial belief that the experiment would be rather quick and easy, and would provide rough values of the masses, which might be helpful in the cyclotron resonance experiments that would determine the same quantities more accurately. In fact our experiment did not prove to be so easy, and the cyclotron resonance experiments have encountered various difficulties, so that at present, these rather imprecise mass measurements are the only ones available. We will show in this paper that the results do exclude certain of the suggested structures and favor others, and that they provide a significant amount of information about the carriers.

In the sections that follow we will first give some background information and describe several models of the ions; we will then present the simplified theory with which we had planned to analyze the data, and extract values of the masses from it. After a presentation of the experimental technique and the data, we will re-examine the theory in the light of the experimental results. We then discuss the conclusions about the nature of the ions that can be drawn from the experiment.

\section{THEORY}

\subsection{Previous Experimental Information-Models of the Ions}

Charged particle mobilities in liquid helium have been measured under a variety of conditions, and by a number of different techniques. We refer the reader to the literature ${ }^{2}$ for these results, which we will not reproduce here, but will require at a later stage of the analysis. The mobility data suggested to a number of workers that the negative carrier was not simply a nearly free electron, and the positive carrier was not a light helium ion (such as $\mathrm{He}^{+}$or $\mathrm{He}_{2}^{+}$). Probably the most relevant features of the mobility data, from this point of view, are: the rather large scattering cross sections ${ }^{3-5}$ implied by the low values of the mobilities, and the fact that the mobility of the negative carrier is less than that of the positive ion.

In 1960, Careri, Fasoli, and Gaeta ${ }^{6}$ reported an interesting experiment, which led them to conclude that the positive ion was a solid cluster of helium atoms polarized around a positive charge, a structure described by Atkins ${ }^{7}$ in 1959 , and that the negative ion was an electron "in a cage, self-trapped by a shell of polarized atoms." Such a structure (which we will call the bubble model) had 
been suggested for positronium in liquid helium ${ }^{8}$ in 1957. Some of the properties of such a negative carrier were calculated by $\mathrm{Kuper}^{9}$ in 1961. A more comprehensive discussion of the properties of the negative carrier in terms of the bubble model has been presented by Springett, Cohen, and Jortner. ${ }^{10}$ There has not by any means been unanimous agreement on the structure. Atkins ${ }^{7}$ suggested quite a different picture of the negative carrier ; Meyer, Davis, Rice, and Donnelly, ${ }^{11}$ in 1962, suggested still another model for each carrier. Experiments on the mobility of photoelectrons in dense, cold helium gas ${ }^{12}$ offered support for the bubble model, as did a hydrodynamic interpretation ${ }^{13}$ of the binding of the negative carrier to vortices. A similar analysis of the binding of the positive carrier to vortices $^{13}$ yielded ion radii in reasonable agreement with Atkins' calculations. The effective masses predicted by the various models differ rather widely; in the case of the negative carrier, published theoretical values vary by five orders of magnitude. Under such circumstances even a rather crude experimental determination of the mass can be very helpful in guiding theoretical thinking.

\subsection{Simplified Theory of the Method}

The dc mobility of a charged particle in a medium only provides information about the ratio of the momentum relaxation time $(\tau)$ of the carrier to its effective mass $\left(M^{*}\right)$. If, however, the mobility can be measured at high frequencies, where $\omega \tau$ is at least comparable to one, then the relaxation time and effective mass can be separately evaluated. This situation obtains because at high frequencies both frictional and inertial effects are important; the drift velocity of the carriers is no longer in phase with the applied electric field, and the mobility contains terms dependent on $\omega \tau$ rather than just $\tau / M^{*}$. These same effects have been used to determine relaxation times and effective masses of carriers in gases ${ }^{14}$ and solids. ${ }^{15}$ The simplest analysis begins with an "equation of motion of the average carrier" in the presence of collisions and an alternating electric field, $\mathscr{E}$, of frequency $\omega$ :

$$
M^{*}\left(d \mathbf{v}_{\mathbf{d}} / d t\right)+M^{*}\left(\mathbf{v}_{\mathbf{d}} / \tau\right)=e \mathscr{E}
$$

Here $\mathbf{v}_{\mathbf{d}}$ is the drift velocity of the ion. The mobility, $\mu$, obtained from Eq. (1) is

$$
\mu(\omega) \equiv \frac{\mathbf{v}_{\mathbf{d}}}{\mathscr{E}}=\frac{e \tau}{M^{*}} \frac{1-i \omega \tau}{1+(\omega \tau)^{2}} \equiv \mu_{r}+i \mu_{i}
$$

its de value is

$$
\mu(0)=e \tau / M^{*}
$$

The quantity of interest, $\omega \tau$, is equal to the ratio of the imaginary to the real part of the mobility. These components can be measured by measuring the change in the imaginary and real parts of the voltage reflection coefficient, $\Gamma$, when a sample of ions is introduced into a microwave reflection cavity. The relationship among 
these quantities for a critically coupled cavity is

$$
\delta \Gamma=\frac{-2 \pi Q_{0}}{\omega} \frac{\int n(\mathbf{r}) e\left(\mu_{r}+i \mu_{i}\right) \mathscr{E}^{2}(\mathbf{r}) d^{3} r}{\int \mathscr{E}^{2}(\mathbf{r}) d^{3} r}
$$

with

$$
\delta \Gamma \equiv \delta \Gamma_{r}+i \delta \Gamma_{i}=C\left(\mu_{r}+i \mu_{i}\right)
$$

Here $Q_{0}$ is the unloaded $Q$ of the cavity; $C$ is a constant independent of $\omega \tau$; $n(\mathbf{r})$ and $\mathscr{E}(\mathbf{r})$ are, respectively, the density of ions and the microwave electric field strength at position $\mathbf{r}$ in the cavity. The integral is extended over the cavity. The ratio $\mu_{i} / \mu_{r}$ can be determined by measuring $\Gamma_{i} / \Gamma_{r}$, without requiring a knowledge of the distribution of ions in the cavity or the sensitivity of the microwave spectrometer. However, the resonant frequency shift $\left(\Gamma_{i}\right)$ proved to be very difficult to measure in our experiment, and a more accurate value of $\omega \tau$ was obtained by determining the distribution of the ions and the sensitivity of the spectrometer, and making an absolute measurement of $\mu_{r}$. The ratio of the $\mathrm{dc}$ mobility to the real part of the microwave mobility can be obtained from Eqs. (2) and (3):

$$
\mu(0) / \mu_{r}(\omega)=1+(\omega \tau)^{2}
$$

We have used both methods to obtain the relaxation time, and therefore the effective mass, as a function of the temperature. Some limitation on the range of applicability of Eq. (1) will be discussed in Section 6.

\section{EXPERIMENTAL APPARATUS}

\subsection{Microwave Spectrometer}

A block diagram of the two-bolometer bridge $x$-band spectrometer ${ }^{16}$ is shown in Fig. 1. The microwave source is an L.F.E. model 814 stabilized oscillator. The microwave power level incident on the cavity is set at $2.7 \mathrm{~mW}$. The cavity is critically coupled to the waveguide with a variable iris, to optimize signalto-noise ratio. This type of spectrometer can be adjusted to measure the change in either the real or the imaginary component of the reflection coefficient by adjusting the phase shifter (Hewlett Packard model X-885 A) in the reference arm of the bridge. The output of the balanced bolometer detector is amplified with a narrow-band amplifier, fed into a phase-sensitive detector and the rectified signal is displayed on a strip-chart recorder.

\subsection{Sample Cavity}

A cross section of the cylindrical cavity, which is filled with liquid helium in the experiment, is shown in Fig. 2. The ions are produced outside the cavity. The ion beam entering the cavity is chopped with an electrical gate that is synchronous with the reference signal fed to the phase-sensitive detector. The cavity 


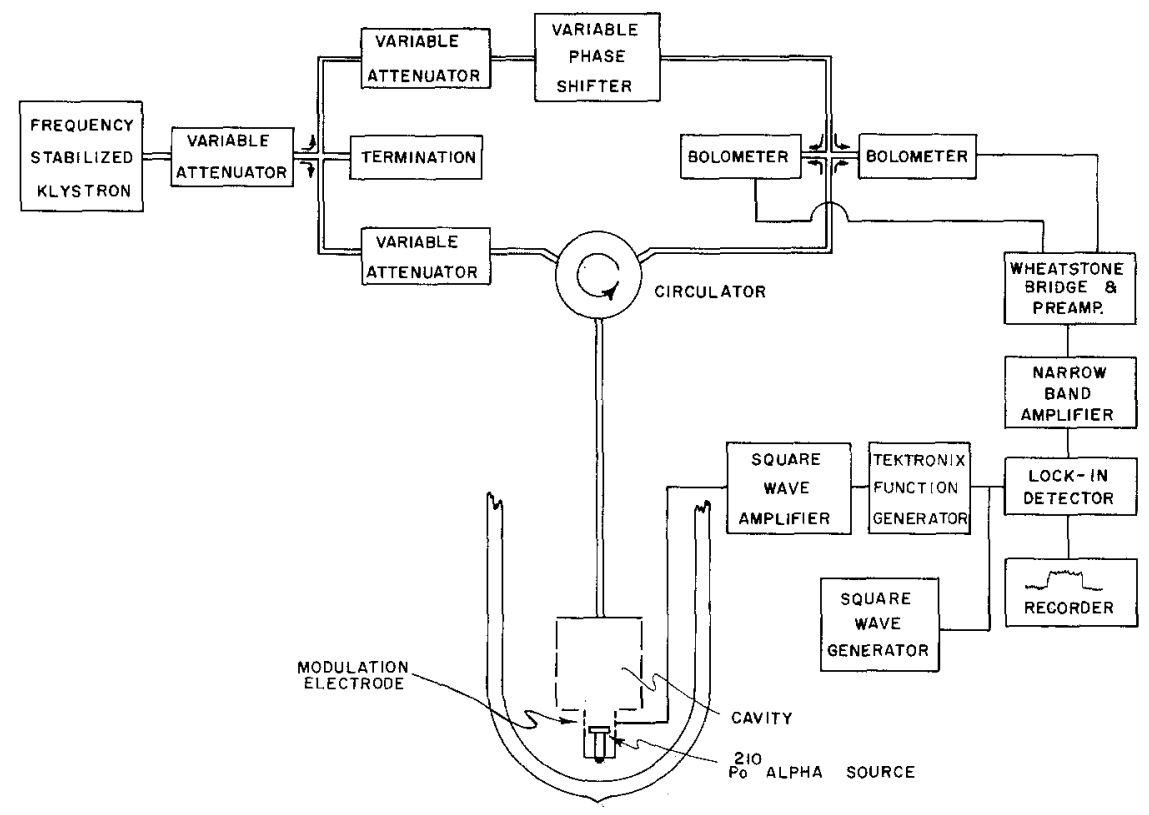

Fig. 1. Block diagram of microwave spectrometer.

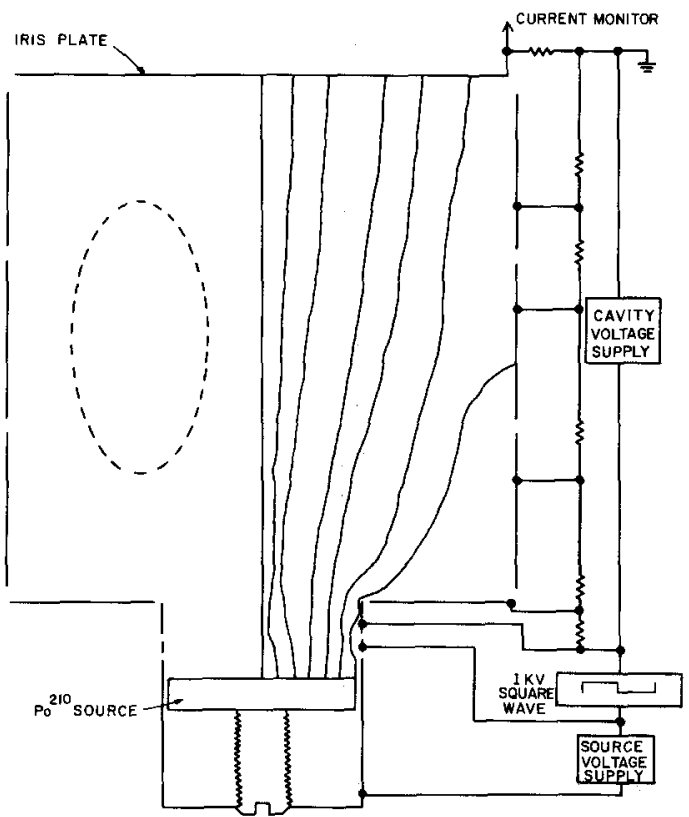

Fig. 2. Cross section of microwave cavity. The square of the microwave electric field inside the dashed curve is larger than half-maximum. The field lines in the right half of the figure represent the trajectories of the ions under the influence of space charge fields for a typical setting of electrode potentials and ion density. 
is operated in the $\mathrm{TE}_{011}$ cavity mode, in which the microwave electric field is entirely azimuthal. In this mode the cavity walls can be constructed with rings, insulated from each other and from the ends of the cavity, without cutting microwave current lines. With this design a de electric field can be applied to control the ion trajectories in the cavity. The dc potentials on the various parts of the cavity (hereafter referred to as the cavity electrodes) can be adjusted so that the ions are focused into regions of large microwave electric field. The position of the coupling iris and the ring construction of the cavity walls eliminate complications from the degenerate $T M_{111}$ mode. The iris plate is insulated from the waveguide and the cavity walls and serves as a current collector, so that the ion current waveform can be monitored on an oscilloscope. The cavity diameter is $4.05 \mathrm{~cm}$ and its length is $4.3 \mathrm{~cm}$; its resonant frequency in the $\mathrm{TE}_{011}$ mode is $9.1 \mathrm{GHz}$ when it is filled with liquid helium. The electrodes are made from hydrogen-fired oxygen-free copper, and are separated by $0.02-\mathrm{cm}$ mylar spacers. The $Q$ of the cavity is somewhat dependent on the alignment of the cavity electrodes, and is approximately 70,000 at liquid helium temperatures.

The dc electric-field configurations inside the sample cavity were studied to determine the distribution of ions. The trajectories of the ions are determined by the vector sum of the applied dc field and a space charge field that is calculable. The applied dc field was mapped in an electrolytic tank with a section of a scaled model of the cavity and the calculated space charge field was added to it. The resultant electric field lines are shown in the right half of Fig. 2 for a typical setting of electrode potentials and ion density. The square of the microwave electric field inside the dashed curve is larger than half-maximum. Voltages are applied to each electrode by a voltage divider located outside the dewar.

\subsection{Ion Source}

A $50-\mathrm{mCi}{ }^{210} \mathrm{Po}$ radioactive source, supplied by Monsanto Research Corporation, is used to produce ionization in the liquid helium, and a source electric field of appropriate sign is applied to obtain the desired species of ion. The polonium is plated on a $1.5-\mathrm{cm}$ diameter brass disk, and is coated with a thin layer of gold to prevent migration of the polonium. This disk is placed $1 \mathrm{~cm}$ from the cavity in an extension that is attached to the bottom of the cavity. The extension has a $1.6-\mathrm{cm}$ inner diameter, and is beyond cutoff for $3-\mathrm{cm}$ microwaves.

The ion beam is modulated by applying a $1-\mathrm{kV}$ peak-to-peak symmetric square wave between two rings that are part of the extension. A square wave of this amplitude is necessary to retard all ions during one half cycle and to pass a sufficiently large ion current during the other. Grids were used in an early version of the apparatus, but were found to be excessively microphonic.

\subsection{Other Apparatus}

The modulation voltage is produced by amplifying a signal from a Tektronix model 161 pulse generator, triggered by a sawtooth voltage from a Tektronix model 162 waveform generator. A Hewlett Packard model 202 low-frequency generator 
provides the square wave reference signal to the phase-sensitive detector and triggers the sawtooth generator (see Fig. 1). The relative phase of the modulating voltage and the reference signal is adjustable. Modulating frequencies of 5,11, 16, $22,27,30$, and $36 \mathrm{~Hz}$ were used, corresponding to the twin-T circuits we had on hand for the narrow band amplifier.

The leads to the cavity electrodes, which must carry several kilovolts through helium vapor, consist of two sets of four nichrome leads each. Each nichrome wire fits snugly into a teflon sleeve. The teflon-insulated wires are inserted into a $\frac{3}{8}$-in. o.d. thin-walled stainless steel tube that is filled with polyurethane "foam in place" resin to prevent the leads from vibrating. An epoxy resin vacuum seal is made outside the dewar between the teflon sleeves and the tube. Any ac voltages on the cavity electrodes will produce forces that will modulate the resonant frequency of the cavity and/or the leakage through the electrodes. To prevent induced voltages from appearing on the cavity electrodes, the leads to the walls and base of the cavity are enclosed in a separate stainless steel tube from the leads carrying the modulating square wave and source voltages, and the shield tubes are grounded. In addition, the leads to the cavity electrodes are capacitively bypassed to ground and the sample cavity is wrapped with a layer of steel wool. The steel wool is grounded and insulated from the cavity with a layer of teflon. The current-monitoring lead from the iris is separately sealed in a 3-mm o.d. grounded silvered glass tube, to maintain a high impedance to ground.

The temperature is measured using an Octoil " $S$ " manometer and the "1958 ${ }^{4} \mathrm{He}$ Scale of Temperatures." A carbon resistor and heater feedback system stabilizes the temperature to within $\pm 0.001 \mathrm{~K}$.

\section{EXPERIMENTAL PROCEDURE}

\subsection{Spectrometer Tuning Procedure}

The spectrometer is sensitive to absorption $\left(\Gamma_{r}\right)$ when the microwaves from the cavity and the reference arm of the bridge are in phase at one of the bolometers. The noise from our spectrometer is predominately dispersive and is a minimum when the instrument is tuned to be sensitive to absorption signals. The spectrometer is tuned to be sensitive to absorption by adjusting the microwave phase shifter for minimum noise. At large microwave power levels a sharp noise minimum exists (the noise increases by a factor of 3 for a $3^{\circ}$ phase error) as a function of the phase of the microwaves in the reference arm of the spectrometer. The spectrometer is tuned to measure dispersion $\left(\Gamma_{i}\right)$ by shifting the reference arm phase by $90^{\circ}$ from the noise minimum.

This tuning procedure was checked by placing the sample cavity in a dc magnetic field of $3000 \mathrm{G}$ with an ac magnetic field superimposed. The ac magnetic field set up eddy currents in the cavity walls that, in the presence of the de field, forced the walls to oscillate. The resulting frequency modulation of the cavity yielded a minimum signal when the spectrometer was tuned to minimum noise. An additional check was made by frequency modulating the cavity in the following 
manner. The ion source shown in Fig. 2 was removed from the cavity extension and a 1.6-cm diameter teflon rod was inserted through the extension, projecting approximately $3 \mathrm{~mm}$ into the cavity. The teflon rod rested on a small loudspeaker that was driven by a Tektronix square wave generator. The resultant signal should be almost purely dispersive $\left(\Gamma_{i}\right)$, since the power factor of teflon is less than $3.7 \times 10^{-4}$ at $10 \mathrm{GHz}$. The minimum in signal due to the oscillating teflon rod coincided with minimum noise to within $1^{\circ}$. Previous use of the spectrometer in electron spin resonance experiments also showed that the minimum noise phase seiting coincided accurately with sensitivy to $\Gamma_{r}$ only.

In this work there is no resonance, and the measured quantity is a dc shift of the recorder trace. Therefore any detected signal must be identified as a real signal or a stray (pickup) signal. At liquid helium temperatures (but not at room temperature), some pickup is usually present, presumably caused by a slight vibration of the source when the square wave is applied. The residual pickup is characterized by the following properties: It is constant in magnitude throughout a given run for a particular set of voltages applied to the electrodes; its magnitude is independent of the modulating frequency; it has a specific phase with respect to the modulating square wave. This phase is determined by studying the false signals under operating conditions, but with the polonium source replaced with a brass disk.

Before taking data a signal from the square wave generator is introduced into the narrow band amplifier and the frequency is adjusted to yield a peak output. This insures that the modulation frequency is identical to the tuned frequency of the narrow band amplifier and eliminates a source of phase shift. At any given temperature the proper voltages are applied to the electrodes, and the source field is set opposite in sign to the field in the cavity; thus no ions enter the cavity. If any signal is detected under these conditions, the phase of the modulating square voltage is adjusted for a null signal. If no pickup is detected, the phase is set at the predetermined phase for null pickup.

Since the phase of the modulating square wave voltage is preset for a null pickup signal, the phase of the true signal (due to the conductivity of the ions) should be adjusted to be $90^{\circ}$ out of phase with the pickup signal. This is accomplished by adjusting the transit time of the ions across the sample cavity. The phase of the true signal is the same as that of the pickup signal when the transit time, $T_{c}$, is much shorter than the period, $T_{m}$, of the modulating square wave voltage. The phase lag of the signal relative to the case for which $T_{c} \ll T_{m}$ is given by

$$
\theta=\pi T_{c} / T_{m}
$$

The prediction of Eq. (7) was confirmed, within experimental error, by working with a sample of positive ions in nitrogen gas at $300 \mathrm{~K}$ and 3-atm pressure, where pick up was negligible at room temperature. The modulating frequency and cavity voltage are adjusted for each mobility so that the transit time is equal to one-half period. The transit time is determined from the ion current waveform.

The ion current is varied by adjusting the source voltage, and at each temperature the signal (the change in recorder output when the source voltage is 
reversed) is recorded for various ion currents. The dispersion data are taken under conditions identical to those at which some absorption data are taken. The only change is the adjustment of the phase shifter in the reference arm of the spectrometer.

\subsection{Spectrometer Sensitivity Determination}

In order to determine the absolute value of the microwave mobility, from an observed deflection of the recorder under a given set of operating conditions, the sensitivity of the spectrometer must be known. A balanced bolometer bridge detector measures the difference in microwave power, $\Delta P$, incident on the two bolometers. The change in $\Delta P$ when a sample of ions is introduced into the sample cavity is given by

$$
\delta(\Delta P)=\operatorname{Re}\left\{-2\left(P_{r} P_{c} R\right)^{1 / 2} e^{i \phi} \delta \Gamma\right\}
$$

Here $P_{r}$ and $P_{c}$ are, respectively, the microwave power in the reference arm of the spectrometer and the microwave power incident on the cavity, $R$ is a number less than unity and accounts for losses in the circulator and isolator and reflections from the bolometer bridge, $\phi$ is the phase at one of the bolometers of the microwaves incident from the reference arm of the bridge relative to the microwaves incident from the cavity arm of the bridge, and $\delta \Gamma$ is given by Eq. (4). The expression for $\delta \Gamma$ involves the density and distribution of ions in the cavity. The only measured parameter relating to the density of ions is the current, $I$, which is collected on the iris plate and measured on an oscilloscope. The ion current can be written as

$$
I=\int_{A} n(\mathbf{a}) e \mu(0) \mathscr{E}_{A} d^{2} a
$$

where the integral is taken over a cross section of area $A$ in the center of the cavity, $n(\mathbf{a})$ is the density of ions at a position a on this surface, and $\mathscr{E}_{A}$ is the magnitude of the dc electric field component parallel to the axis of the cavity (assumed independent of a). Combining Eqs. (4), (8), and (9) we have

$$
\delta(\Delta P)=\operatorname{Re}\left\{4 \pi(Q / \omega)\left(P_{r} P_{c} R\right)^{1 / 2} e^{i \phi} \frac{\left(\mu_{r}+i \mu_{i}\right)}{\mu(0)} \frac{I B}{A \mathscr{E}_{A}}\right\}
$$

where $B$ is defined as

$$
B \equiv \frac{\int n(\mathbf{r}) \mathscr{E}^{2}(\mathbf{r}) d^{3} r}{\int \mathscr{E}^{2}(\mathbf{r}) d^{3} r} / \frac{\int_{A} n(\mathbf{a}) d^{2} a}{A}
$$

This dimensionless quantity depends on the ratio of the applied dc field in the cavity to the space charge field, and on the configuration of voltages applied to the cavity electrodes. The density of ions as a function of position was determined from field plots, such as the right half of Fig. 2, and $B$ was determined by graphical 
integration. If the density of ions were constant, $B$ would be equal to unity. In the present experiment the ions were focused into regions of large microwave electric field and $B$ was typically $1.35 \pm 0.15$. The maximum density of ions we could attain was approximately $10^{8} \mathrm{~cm}^{-3}$ at a cavity voltage of $3000 \mathrm{~V}$. This limitation on density was a result of space-charge fields, which drove any additional current entering the cavity to the walls. The wall current was occasionally measured under operating conditions, and was taken into account in determining $B$.

The bolometers are used as two arms of a Wheatstone bridge, whose output is transformer-coupled into a preamplifier. The ac open-circuit output of the Wheatstone bridge, $\delta V_{0}$, is

$$
\delta V_{0}=I_{0} S_{0} \delta(\Delta P)
$$

where $I_{0}$ is the dc bias current through the bolometers and $S_{0}$ is the sensitivity of the bolometers. The signal, $S$, displayed on the chart recorder is

$$
S=F G \delta V_{0}
$$

Here $F$ is the first Fourier coefficient of $\delta V_{0}(t) ; G$ is the ratio of the dc chart recorder deflection (in millivolts) to the ac peak-to-peak input to the preamplifier, and depends on the modulation frequency.

The first Fourier coefficient was calculated, as a function of the ratio of the transit time to the period of the modulation frequency. For $T_{c} / T_{m}>0.5$, the coefficient decreases because a second pulse of ions enters the cavity before the first pulse leaves; thus the cavity is never flushed of ions. The time dependence of the signal was analyzed under the idealized condition of a beam of ions of constant density moving through the cavity at a uniform velocity. For $T_{c} / T_{m} \ll 1$ the signal $\delta V_{0}(t)$ is a square wave; it approaches a sine wave for $T_{c} / T_{m}>0.6$.

Combining Eqs. (10), (12), and (13) and using (2) and (3) to write the mobilities in terms of $\omega \tau$, we have

$$
S / I=\operatorname{Re}\left\{K\left(\mu_{r}+i \mu_{i}\right) e^{i \phi} / \mu(0)\right\}=\operatorname{Re}\left\{K(1-i \omega \tau) e^{i \phi} /\left[1+(\omega \tau)^{2}\right]\right\}
$$

with

$$
K \equiv F G I_{0} S_{0} 4 \pi(Q / \omega)\left(P_{r} P_{c} R\right)^{1 / 2} B /\left(A \mathscr{E}_{A}\right)
$$

Each of the parameters in Eq. (15) can be either measured directly or calculated, so that the spectrometer sensitivity, $K$, can be determined for a given set of operating conditions. All data are referred to a standard set of conditions, for which

$$
K=(1.67 \pm 0.3) M \Omega
$$

\section{EXPERIMENTAL DATA}

\subsection{Method of Analysis}

At a given temperature absorption measurements are taken for various ion currents and operating conditions. A weighted average of the signal-to-ion current 
ratio for various ion currents is determined under a given set of operating conditions. Since noise and the possibility of a stray signal limit the reliability of each measurement, a weight is assigned to each measurement. In order to compare signals ali $S / I$ ratios are referred to a standard set of operating conditions. This comparison involves the applied dc field in the cavity, microwave power incident on the cavity, gain of the amplifiers (which depends on the modulation frequency and the input attenuation), and the $Q$ of the cavity, which varied from run to run. Corrections are also made for variations in the first Fourier coefficient of the signal waveform and the phase of the modulation if $T_{c} / T_{m} \neq \frac{1}{2}$, the phase having been preset to eliminate stray signals. Dispersion measurements are taken and analyzed using the same procedure.

TABLE I

Absorption Data

\begin{tabular}{|c|c|c|c|c|}
\hline $\begin{array}{c}T \\
(\mathrm{~K})\end{array}$ & $\begin{array}{c}\mu(0) \\
\left(\mathrm{cm}^{2} \mathrm{~V}^{-1} \mathrm{sec}^{-1}\right)\end{array}$ & $\begin{array}{c}S / I \\
(\mathrm{~K} \Omega)\end{array}$ & \multicolumn{2}{|l|}{$M^{*} / m_{\mathrm{He}}$} \\
\hline \multicolumn{5}{|c|}{ Positive Ions } \\
\hline 1.295 & 0.933 & $20 \pm 6$ & $41(+8$ & $-5)$ \\
\hline 1.320 & 0.822 & $41 \pm 10$ & $32(+5$ & $-3)$ \\
\hline 1.330 & 0.780 & $40 \pm 11$ & $34(+6$ & $-4)$ \\
\hline 1.333 & 0.764 & $41 \pm 11$ & $35(+6$ & $-4)$ \\
\hline 1.348 & 0.716 & $41 \pm 7$ & $37 \pm 3$ & \\
\hline 1.360 & 0.678 & $58 \pm 23$ & $33(+9$ & $-6)$ \\
\hline 1.434 & 0.496 & $64 \pm 11$ & $42(+5$ & $-3)$ \\
\hline 1.444 & 0.484 & $77 \pm 23$ & $391+8$ & $-5)$ \\
\hline 1.460 & 0.448 & $96 \pm 16$ & $38(+4$ & $-3)$ \\
\hline 1.476 & 0.426 & $86 \pm 26$ & $42(+9$ & $-6)$ \\
\hline 1.523 & 0.355 & $131 \pm 19$ & $40(+4$ & $-3)$ \\
\hline 1.549 & 0.324 & $150 \pm 26$ & $41(+5$ & $-3)$ \\
\hline 1.616 & 0.260 & $176 \pm 22$ & $47 \pm 3$ & \\
\hline 1.645 & 0.239 & $251 \pm 58$ & $42(+7$ & $-5)$ \\
\hline 1.667 & 0.224 & $260 \pm 33$ & $44 \pm 3$ & \\
\hline 1.732 & 0.186 & $258 \pm 45$ & $53(+6$ & $-5)$ \\
\hline 1.896 & 0.123 & $730 \pm 100$ & $38(+6$ & $-4)$ \\
\hline 1.916 & 0.118 & $373 \pm 69$ & $67(+9$ & $-7)$ \\
\hline 2.043 & 0.090 & $525 \pm 115$ & $68(+13$ & $-9)$ \\
\hline 2.168 & 0.059 & $535 \pm 180$ & $103(+33$ & $-21)$ \\
\hline \multicolumn{5}{|c|}{ Negative Ions } \\
\hline $1.732^{a}$ & 0.140 & $84 \pm 57$ & $130(+100$ & $-30)$ \\
\hline $1.732^{a}$ & 0.140 & $132 \pm 80$ & $100(+65$ & $-20)$ \\
\hline 1.797 & 0.119 & $150 \pm 115$ & $112(+128$ & $-32)$ \\
\hline 1.841 & 0.109 & $174 \pm 110$ & $113(+81$ & $-27)$ \\
\hline 1.916 & 0.094 & $180 \pm 95$ & $128(+65$ & $-28)$ \\
\hline \multicolumn{5}{|c|}{ Positive Ions-Helium Gas } \\
\hline 4.2 & $0.1 \pm .02$ & $410 \pm 90$ & $75 \pm 20$ & \\
\hline
\end{tabular}

${ }^{a}$ The two sets of measurements at $1.732 \mathrm{~K}$ differ only in voltage configuration applied to the cavity electrodes. 
Errors were assigned to allow for the following uncertainties: The measurement of the unloaded $Q$ of the cavity; admixture of the imaginary and real components of the reflection coefficient when the bridge is not precisely adjusted; the modulation phase; the ion current; statistical fluctuations in the signal; and an estimate of possible pickup. All errors quoted are at a 50 percent confidence level. The total fractional error, $p$, is computed by using the expression

$$
p^{2}=\sum_{i}\left(p_{i}\right)^{2}+\sum_{\substack{i j \\ i \neq j}}\left(p_{i}\right)^{2}\left(p_{j}\right)^{2}
$$

where $p_{i}$ and $p_{j}$ are individual fractional probable errors.

We present our absorption data in Table I. The signal-to-ion current ratio is an average value referred to standard operating conditions.

\subsection{Reduction of Data}

\subsubsection{Positive Ions}

In Fig. 3 the ratio of the ion current $(I)$ to the signal $(S)$ is plotted against the square of the dc mobility. From Eq. (14) it can be observed that this is a plot of $K^{-1}\left[1+(\omega \tau)^{2}\right]$ vs $\left(e / M^{*} \omega\right)^{2}(\omega \tau)^{2}$ with an intercept at $K^{-1}$ that was determined in the previous section to be $0.6 \pm 0.1 \mu$ mho. If we assume a temperature-independent effective mass, the curve should be a straight line with a slope $D$, given by

$$
D=K^{-1}\left(M^{*} \omega / e\right)^{2}
$$

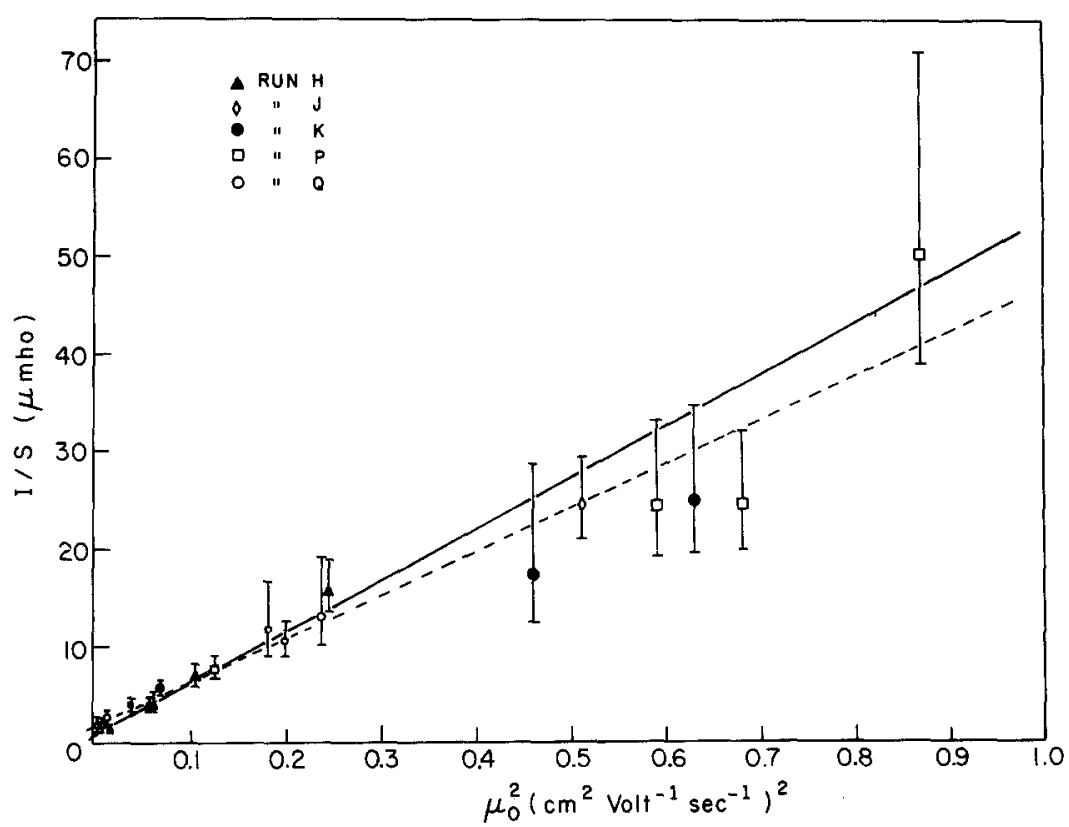

Fig. 3. Ratio of the ion current to the signal vs the square of the de mobility for positive ions. The solid and dashed lines are discussed in the text. 
The dashed line in Fig. 3 represents a least-squares fit to the data and corresponds to an effective mass of 23 helium masses and an intercept at $1.55 \mu \mathrm{mho}$; the latter is in serious disagreement with the value of $K$ given in Section 4. Assuming our sensitivity determination to be correct and regarding only the slope as a free parameter yields the solid line of Fig. 3, which corresponds to an effective mass of 39 helium atom masses.

In Fig. 4 the ratio of the effective mass to the mass of a helium atom as determined from the ratio $\mu_{i} / \mu_{r}$, is plotted against the temperature. The large errors assigned to the data result from the presence of pickup, which is difficult to eliminate when the apparatus is dispersion-tuned. These data suggest that the effective mass may be temperature-dependent, which would also explain the discrepancy in the intercept in Fig. 3.

If such a temperature dependence exists, we can determine $\omega \tau$ by measuring $\Gamma_{r}$ at each temperature and using Eq. (14). We then have

$$
M^{*}=\frac{e}{\omega \mu(0)} \omega \tau=\frac{e}{\omega \mu(0)}\left(\frac{K I}{S}-1\right)^{1 / 2}
$$

The effective mass determined by this method is given in Table I and in Fig. 5 as a function of the temperature. We will show in Section 6 that its increase with increasing temperature is to be expected.

\subsubsection{Negative Ions}

We were able to collect data only in a very narrow temperature range for the negative ions. Below $1.7 \mathrm{~K}$ the negative ion currents were unusably small. This reduction in current is probably caused by the trapping of negative ions in turbulence ${ }^{17}$ that may be present near our high-activity polonium source. Above $2.1 \mathrm{~K}$ the negative ion mobility is so low that in the highest available electric fields the ions cross the cavity in a time longer than one-half period of our lowest modulation frequency $(5 \mathrm{~Hz})$. In addition there was generally a large amount of drift when negative ion signals were under study. No dispersion data are used in analyzing the negative ion effective mass. In Fig. 5, quantity $M^{*}=[e / \omega \mu(0)] \times$ $[K(I / S)-1]^{1 / 2}$ is plotted as a function of the temperature for negative ions as well. The indicated effective mass in this temperature range is approximately 100 to 200 helium masses.

\subsubsection{Positive Ions in Helium Gas}

The $\mathrm{dc}$ mobility of the positive ion in helium gas at the normal boiling point ( $4.2 \mathrm{~K}$ and $1 \mathrm{~atm}$ ) has been measured ${ }^{18}$ and is equal to $0.1 \pm 0.02 \mathrm{~cm}^{2}$ volt $^{-1} \mathrm{sec}^{-1}$. We have taken one measurement of the real part of the microwave mobility in the NBP gas. For this experiment a copper container filled with liquid helium was mounted on the waveguide above the cavity. Liquid helium was also placed in the dewar beneath the cavity; the liquid was in contact with the cavity extension. The gas in the cavity should thus have been nearly at its normal boiling point. Unfortunately, the cavity was very stable for only one measurement. Subsequently, the resonant frequency of the cavity drifted and random frequency modulation of the 


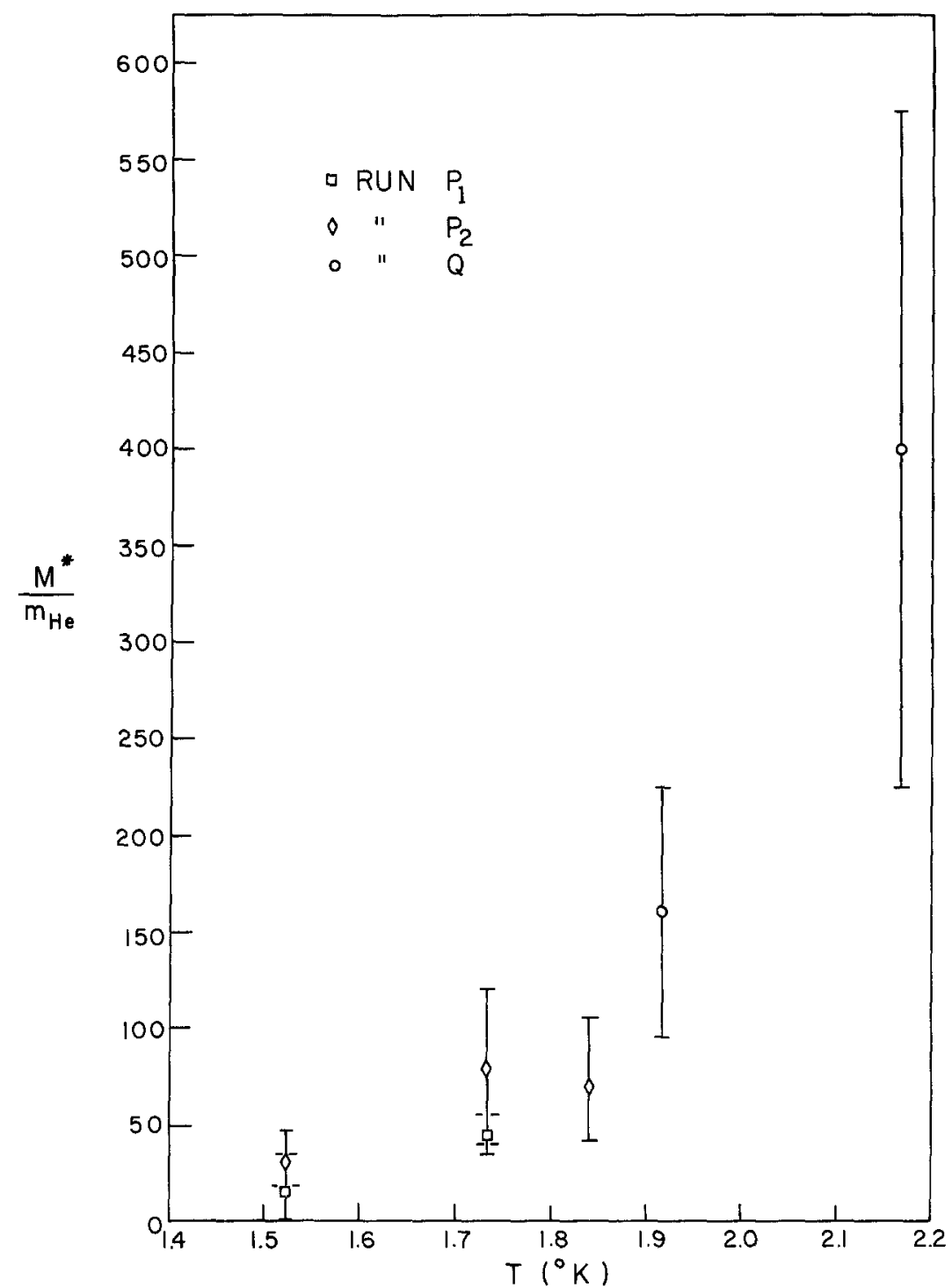

Fig. 4. Positive ion effective mass as determined from the ratio $\mu_{i} / \mu_{\mathrm{r}}$ vs temperature, neglecting viscous effects. Runs $P_{1}$ and $P_{2}$ differ only in voltage configuration applied to the cavity electrodes.

cavity, perhaps because of the boiling helium, set in; we were not able to regain stable conditions in this run or in a subsequent run. A redesign of the equipment is needed for further gas measurements.

Our single result indicates a value of

$$
M^{*}=[e / \omega \mu(0)][K(I / S)-1]^{1 / 2}=75 \pm 20 m_{\mathrm{He}}
$$




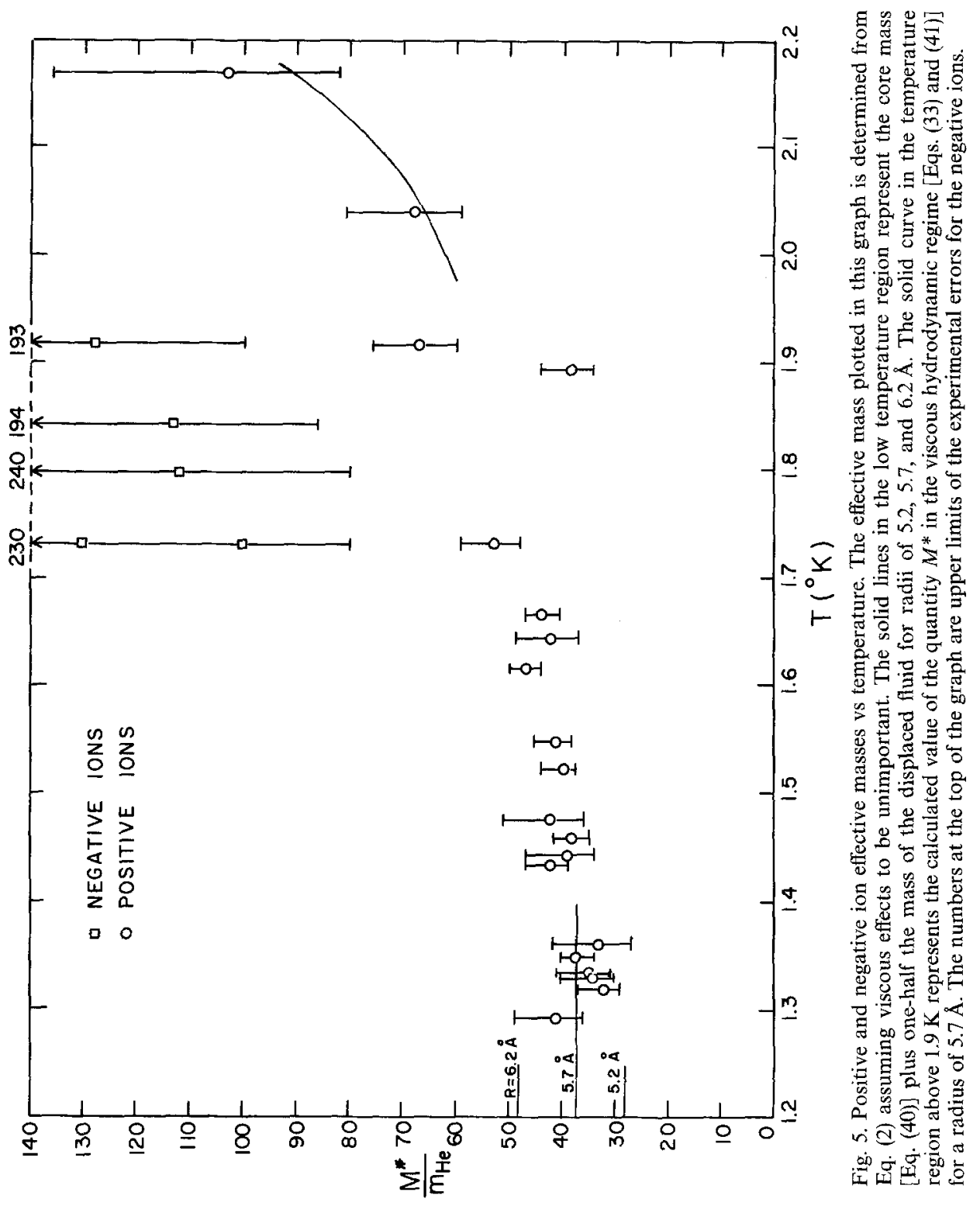




\section{REFINED THEORY}

\subsection{Hydrodynamic Effects}

The experimental observation (Fig. 5) that the effective mass of the positive ion in He II rises as the lambda point is approached suggests that viscous effects in the normal fluid component are making an important contribution. Under such circumstances, as we will show below, Eq. (1) can not be used to analyze the data. The reason is that our method depends on a comparison of a measurment at zero frequency (the dc mobility) and one performed at a high frequency. In Eq. (1) it is assumed that the inertial term $\left(M^{*}\right)$ is independent of frequency and that the damping force is proportional to the velocity. Neither of these statements is quite correct for an object oscillating in a viscous fluid. The equation of motion of a rigid sphere in a viscous incompressible fluid is a known result of classical hydrodynamics. ${ }^{19}$ In our case, we can take into account contributions from both the normal and superfluid components by writing the equation in the form

$$
\mathbf{F}=e \mathscr{E}=a(\omega) \mathbf{v}_{\mathbf{d}}+m^{*}(\omega) \mathbf{v}_{\mathbf{d}} / d t
$$

with

$$
\begin{gathered}
a(\omega)=6 \pi \eta R(1+R / \delta)=a(0)(1+R / \delta) \\
m^{*}(\omega)=M_{s}+\frac{1}{2} \rho V\left(1+\frac{9}{2} \frac{\rho_{n}}{\rho} \frac{\delta}{R}\right)
\end{gathered}
$$

and

$$
\delta=\left(2 \eta / \rho_{n} \omega\right)^{1 / 2}
$$

Here $\eta$ is the normal fluid viscosity; $M_{s}, R$, and $V$ are the mass, radius, and the volume of the sphere respectively; $\rho_{n}$ and $\rho$ are the normal and total fluid densities, and $\delta$ is the "penetration depth." The latter quantity is the distance in which transverse waves generated in the viscous medium by the oscillating sphere are damped. The solution of Eq. (21) is analogous to the solution of Eq. (1). The zerofrequency solution yields Stokes' law

$$
\mu(0)=e / a(0)=e / 6 \pi \eta R
$$

The equation of motion for a spherical cavity oscillating in a viscous, incompressible fluid has also been derived. The approach to the problem is identical to that of the rigid sphere case except for a change in the boundary conditions at the surface of the sphere. The boundary conditions at the surface of the cavity are: The normal velocity component of the fluid vanishes in a frame of reference in which the cavity is at rest; the tangential component of the viscosity stress tensor of the fluid vanishes. The equation of motion is given by Eq. (21) with

$$
a_{c}(\omega)=4 \pi \eta R\left[\frac{1+\frac{4}{3}(R / \delta)+\frac{2}{3}\left(R^{2} / \delta^{2}\right)}{1+\frac{2}{3}(R / \delta)+\frac{2}{9}\left(R^{2} / \delta^{2}\right)}\right]
$$


and

$$
m_{c}^{*}(\omega)=\frac{1}{2} \rho V\left[1+\frac{2 \rho_{n} \delta / \rho R}{1+\frac{2}{3}(R / \delta)+\frac{2}{9}\left(R^{2} / \overline{\delta^{2}}\right)}\right]
$$

The zero frequency solution yields the Rybczynski-Hadamard formula for flow around an empty cavity ${ }^{20}$

$$
\mu(0)=e / 4 \pi \eta R
$$

At a finite frequency we obtain for both cases.

$$
\begin{gathered}
\mu(\omega)=\frac{[e / a(\omega)]\left[1-i \omega m^{*}(\omega) / a(\omega)\right]}{1+\left[\omega m^{*}(\omega) / a(\omega)\right]^{2}}=\mu_{r}+i \mu_{i} \\
\left|\mu_{i} / \mu_{r}\right|=\omega m^{*}(\omega) / a(\omega)
\end{gathered}
$$

The measured quantity defined by Eq. (14) is now given by

$$
\frac{S}{I}=K \frac{\mu_{r}}{\mu(0)}=\frac{K a(0) / a(\omega)}{1+\left[\omega m^{*}(\omega) / a(\omega)\right]^{2}}
$$

The quantity $M^{*}$ plotted in Fig. 5 in the temperature range in which Eq. (21) is applicable is obtained after substitution of Eq. (25) into Eq. (31) and rearranging as

$$
M^{*}=\left(\frac{e}{\omega \mu(0)}\right)\left(K \frac{I}{S}-1\right)^{1 / 2}=\left\{\left[\frac{a(\omega)}{a(0)}-1\right]\left[\frac{e}{\omega \mu(0)}\right]^{2}+\frac{\left[m^{*}(\omega)\right]^{2} a(0)}{a(\omega)}\right\}^{1 / 2}
$$

For the case of the rigid sphere this expression simplifies to

$$
M^{*}=\left\{\frac{R}{\delta}\left[\frac{e}{\omega \mu(0)}\right]^{2}+\frac{\left[m^{*}(\omega)\right]^{2}}{1+R / \delta}\right\}^{1 / 2}
$$

We emphasize that although the quantity $M^{*}$ is presented in Fig. 5, the quantity of interest is $m^{*}(\omega)$ in the limit in which the penetration depth vanishes. The frequency-dependent effective mass $m^{*}(\omega)$ can be obtained if the range of applicability of Eq. (21) is determined. The penetration depth can be calculated and $R$ can be determined by a best fit to the data.

\subsection{Range of Applicability of the Equations}

Measurements of dc mobilities of ions in liquid helium yield considerable information as to the temperature region in which viscous flow in the normal fluid is important. A plot of dc mobility vs temperature for both species is shown in Fig. 6 in the temperature range from $1.8 \mathrm{~K}$ to $4.2 \mathrm{~K}{ }^{21}+$ The solid curves in the high temperature region are Stokes' law mobilities for the positive and negative

$\dagger$ We have normalized all mobilities to the values of Reif and Meyer, who quote an absolute accuracy of approximately $5 \%$. Values obtained by Northby were increased by $10 \%$ and values from Dahm et al. and Kuchnir were increased by $15 \%$ to coincide with the data of Reif and Meyer. This suggests that the values of Reif and Meyer may be high. The Stokes' radii and the value of the effective mass in the low temperature regime are inversely proportional to the dc mobility and would have to be adjusted accordingly. 


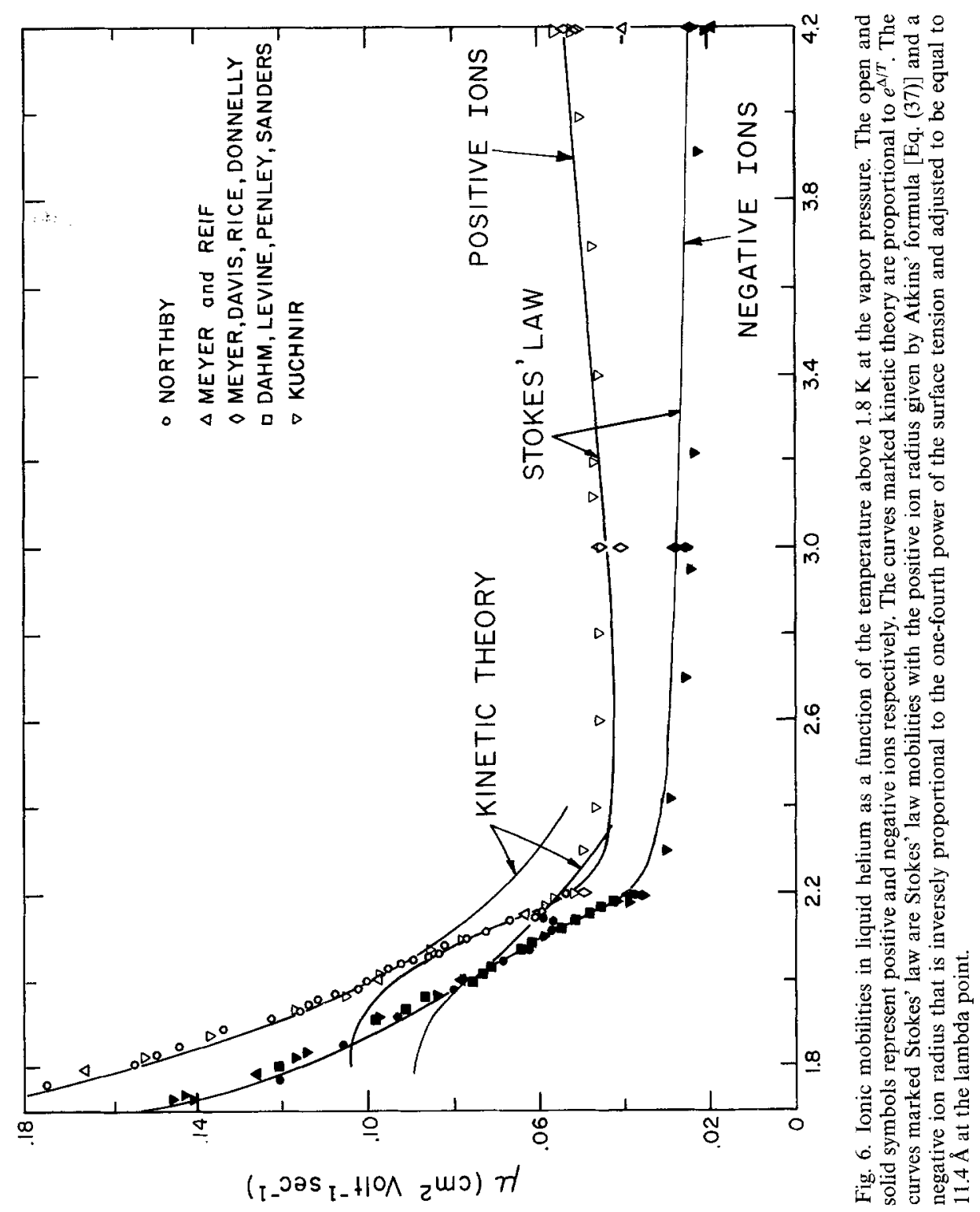


ions with $R_{+}$given by Atkins' formula [Eq. (37), Section 7] and $R_{-}$inversely proportional to the one-fourth power of the surface tension and adjusted to be equal to $11.4 \AA$ at the lambda point. $R_{+}$and $R_{-}$are the radii of the positive and negative ions, respectively. Values for the viscosity were taken from Tough et al. ${ }^{22}$ in the helium II region and from Taylor and Dash ${ }^{23}$ in helium I. Values of the viscosity of liquid helium given in the literature are not in complete agreement. $\dagger$ The solid curves in the low temperature region represent the kinetic theory mobility, which is inversely proportional to the number density of rotons. The mobilities above $2 \mathrm{~K}$ obey Stokes' formula and below $2 \mathrm{~K}$ can be interpreted in terms of kinetic theory. The sharp break between the two regions at $2 \mathrm{~K}$ occurs because the roton mean free path is strongly temperature-dependent. At $2 \mathrm{~K}$ the roton-roton mean free path ${ }^{24}$ is comparable to $R_{+}$. We will assume Eq. (21) to be valid for $T>2 \mathrm{~K}$. We do not know how to calculate the contribution of the elementary excitations to the effective mass below $2 \mathrm{~K}$.

\section{INTERPRETATION OF DATA-MODELS OF THE ION}

\subsection{Positive Ions in Liquid Helium}

Our data on positive ions in liquid helium are far more extensive and reliable than the data on the negative carrier in He II or the positive one in helium gas. Rather definite conclusions can be drawn regarding the structure of the positive ion. The measurements plotted in Fig. 5 show clearly that the measured quantity $\left(M^{*}\right)$ is of the order of tens of helium masses, and that its magnitude rises as the temperature is raised toward the lambda point. The considerations of the last section furthermore show that such a rise is to be expected, and that it is associated with a contribution to the mass from viscous flow in the normal fluid component, whose density is very small at low temperatures, but rises as the lambda point is approached. Models in which the effective mass is of the order of the electron mass (e.g., hole conduction) or of the order of one or two helium masses (e.g., a light ion interacting weakly with the surrounding fluid) are thus clearly excluded. The electrostrictive complex model (variously called the "snowball" or "iceberg", model) of Atkins does predict masses of the correct general magnitude, and we will examine in some detail the extent to which the data and the model are consistent.

The model begins with the observation that in the field of a point charge the surrounding helium atoms will become polarized and therefore experience an attractive force toward the charge. Associated with this volume force, there will be a pressure gradient, so that the helium near the charge will be compressed. At some distance from the charge the local pressure will exceed the melting pressure. If this distance is macroscopic, the helium will presumably be solid inside and liquid outside. In fact, this characteristic distance comes out about $6 \AA$, and it is questionable whether one can apply these classical continuum ideas on such a scale. Nonetheless, if the model is taken very literally, the helium density can be

\footnotetext{
$\dagger$ The viscosity values which disagree with those used are smaller by as much as $15 \%$. These values give a larger Stokes' radius. The Stokes' radius is also dependent on the normalization of the mobility values obtained by various workers.
} 
calculated as a function of position near the ion and a definite prediction can be made regarding the effective mass to be expected. There will be a mass contribution from the solid core, a hydrodynamic contribution from the surrounding fluid, and perhaps an extra contribution from the high-density fluid surrounding the solid core. We will now explore such a model quantitatively. Much of what we do in this section repeats Atkins' work, although we find our treatment somewhat simpler.

A polarizable atom in the presence of an electric field gradient will experience a force

$$
\mathbf{F}=\operatorname{grad} \frac{1}{2} \alpha \mathscr{\delta}^{2}
$$

where $\alpha$ is the atomic polarizability. The pressure gradient in a polarizable fluid in the vicinity of an ion is given by

$$
\operatorname{grad} P=n \operatorname{grad} \frac{1}{2} \alpha \mathscr{E}^{2}=\operatorname{grad}\left(\frac{1}{2} n \alpha e^{2} / \varepsilon^{2} r^{4}\right)-\left(\frac{1}{2} \alpha e^{2} / \varepsilon^{2} r^{4}\right) \operatorname{grad} n
$$

Here $n$ is the number density of atoms, $\varepsilon$ is the dielectric constant, and $r$ is the distance from the ion. In the liquid the gradient of $n$ is small and the pressure can be written as

$$
P-P_{0} \cong \frac{1}{2} n \alpha e^{2} / \varepsilon^{2} r^{4}
$$

where $P_{0}$ is the external pressure applied to the liquid. At some distance $R$ from the ion the pressure will equal the melting pressure, and at closer distances the helium will be solid. $R$ is the radius of the ion and is given by

$$
R=\left[\frac{n \alpha e^{2}}{2 \varepsilon_{m}^{2}\left(P_{m}-P_{0}\right)}\right]^{1 / 4}
$$

where $\varepsilon_{m}$ and $P_{m}$ are respectively the dielectric constant and pressure along the melting curve. In computing $R$ as a function of temperature we have taken $n$ to be the average of the densities at the vapor pressure and the melting pressure. We have not taken the unknown liquid-solid surface tension into account in calculating the radius of the ion; it can be neglected if it is less than $0.1 \mathrm{erg} / \mathrm{cm}^{2}$. One would certainly expect the liquid-solid surface tension to be less than the liquid-vapor surface tension.

The value of $R$ given by Eq. (37) ranges from $4.2 \AA$ at the boiling point to $6.7 \AA$ at absolute zero. This is too large by approximately $1 \AA$ to fit our data. It is in fair agreement with the value $6.44 \pm 0.10 \AA$ obtained by Parks and Donnelly ${ }^{13}$ from the binding energy of positive ions to vortices. Equation (37) was used to calculate the Stokes mobility for positive ions shown in Fig. 6.

The mass of the core has been calculated for radii of 5 and $6 \AA$. We have assumed the pressure inside the core to be given by

$$
P=A_{0} \frac{n}{\varepsilon^{2} r^{4}}-\int_{R}^{r} A_{0} \frac{\operatorname{grad} n \cdot \mathbf{d} \rho}{\varepsilon^{2} \rho^{4}}
$$


where $A_{0}$ is determined by

$$
P_{m}=A_{0} \frac{n}{\varepsilon^{2} R^{4}}
$$

and is not necessarily equal to $\frac{1}{2} \alpha e^{2}$. The density is known as a function of the pressure and $\operatorname{grad} n$ is determined from a plot of $n$ vs $P$ to give a self-consistent solution. The mass of the core, obtained by graphically integrating the density over the volume of the core, was found to be approximately

$$
M_{s}=1.7 \rho \frac{4 \pi}{3} R^{3}
$$

for both radii. Here $\rho$ is the density of the liquid at its vapor pressure.

In analyzing the data we assume the model described above to be valid for the positive ion and assume the electrostrictive contribution to the effective mass outside the solid core to be negligible. We do not agree with the large estimates of the electrostrictive contribution which some authors have used. Zimmermann, ${ }^{25}$ using the theory of a classical ideal fluid, has estimated the electrostrictive contribution to the effective mass of a point charge in liquid helium, assuming no solidification near the charge, to be a few helium atomic masses. We expect the electrostrictive contribution of the fluid outside the solid core to be less than the case with no solid core and and we have neglected it. Combining Eqs. (23) and (40) we have

$$
m^{*}(\omega)=\rho V\left(2.2+9 \rho_{n} \delta / 4 \rho R\right)
$$

The solid curve in Fig. 5 in the temperature region above $1.9 \mathrm{~K}$ represents Eqs. (33) and (41) with a radius of $5.7 \AA$. If we assume viscous effects to be unimportant in the temperature region below $1.4 \mathrm{~K}$, then the experimental quantity, $M^{*}$, plotted in Fig. 5 represents the effective mass. The solid curves shown in the low temperature region for various radii represent the core masses [Eq. (40)] plus one-half the mass of the displaced fluid. This is equal to the low temperature limit of the frequency-dependent mass $m^{*}(\omega)$ [Eq. (41)]. Equation (37) predicts too large a radius in both regimes.

In addition to the errors shown in Fig. 5 there is a $\pm 9 \%$ error in the value for the effective mass due to uncertainties in the sensitivity of the spectrometer, and an additional $\pm 10 \%$ error due to uncertainties in the absolute value of the mobilities. Accordingly, we quote our value of the effective mass at low temperatures as $m_{+}^{*}=(37 \pm 11) m_{\mathrm{He}}$, corresponding to a radius $R_{+}=(5.7 \pm 0.6) \AA$.

\subsection{Negative Ions in Liquid Helium}

The negative carrier in liquid helium has received more theoretical and experimental attention than the positive one, whereas our data are much more limited in quantity and quality. We will therefore give only a brief discussion of the probable structure of the carrier and the implications of our data. 
There is now a body of evidence indicating that the bubble model of the negative carrier probably gives a fairly good description of its properties. In this model the electron's wave function is concentrated in a spherical cavity in the liquid helium. The equilibrium radius can be calculated by balancing the electron's zero-point pressure against the pressure exerted by the surrounding helium on the wall of the cavity. There is not complete agreement on the method of calculating these pressures, so that there is a spread of theoretical values, ranging from approximately 12 to $20 \AA$ in the literature. Experimental information bearing on this quantity has also yielded values spread over a rather large range, from 14 to $21 \AA$.

Since there is some theoretical reason to believe that the effective mass of a bubble electron should be given by the classical hydrodynamic expression, ${ }^{26}$ $m_{-}^{*}=\frac{1}{2} \rho V$, we may use the rather imprecise data of Fig. 5 to extract a value of the carrier radius. Such an interpretation leads to the value $R_{-}=13.5(+3,-1) \AA$. If viscous effects are assumed to be important $\left[m^{*}(\omega)\right.$ calculated from Eq. (32)] the radius is larger by $\frac{1}{2} \AA$.

Furthermore, as already noted, mobility values in $\mathrm{He} \mathrm{I}$ and in $\mathrm{He}$ II near the lambda point can be calculated from viscous hydrodynamics. When such a calculation is performed for positive ions, the radius implied by the mobility is in satisfactory agreement with the values inferred from the effective mass, from trapping on vortex lines, and from the electrostriction model. When the same type of analysis is performed for the negative carrier (see Fig. 6) the radius derived from the mobility in the viscous flow region at $2.0 \mathrm{~K}$ is $12.5 \pm 1.0 \AA$. This value is reasonably consistent with the value we obtain from the effective mass, but is rather small compared to values derived from ion trapping, ${ }^{13}$ from photoejection, ${ }^{27}$ and from the theory of the bubble model.

\subsection{Positive Ions in Helium Gas}

The high value of the carrier mass deduced in the previous section for positive ions in saturated helium vapor at the normal boiling point suggests that some type of massive complex forms on the ion. A possibility, considered and rejected in the interpretation of electron mobility measurements in helium vapor, ${ }^{28}$ was the formation of liquid droplets on the charged particle. In the case of the electron the high zero-point energy of the particle would prevent it from being bound stably to such a droplet. Since this objection does not apply in the case of the positive ion, we will explore in this section the possibility that the mobility and mass data can be interpreted as due to a droplet of liquid nucleated on the charged particle. The Gibbs-Thomson equation relating the equilibrium radius of a charged droplet to the pressure takes on a particularly simple form when the pressure equals the saturated vapor pressure. We have

$$
R^{3}=n \alpha e^{2} / 4 \gamma
$$

where $n, \gamma$, and $\alpha$ are the number density and surface tension of the liquid drop and the atomic polarizability. Inserting for $n$ and $\gamma$ the values for the liquid at the normal 
boiling point and for $\alpha$ the value $0.201 \times 10^{-24} \mathrm{~cm}^{3}$ yields a predicted droplet equilibrium radius of $12.6 \AA$. Since this radius is fairly small compared to the atomic mean free path in the vapor, it seems reasonable to assume that there is not a hydrodynamic flow pattern in the surrounding gas, and to analyze the motion from the point of view of kinetic theory. Thus the effective mass should be just that of the droplet, and the mobility can be calculated from Langevin theory. The measured mass then implies a radius of $10 \pm 1 \AA$ and the mobility a radius of $12 \pm 1 \AA$. In the mass calculation, as in Eq. (42), we have neglected compression (and possible solidification) at the core of the droplet, but the errors are not expected to be large.

\section{CONCLUSIONS}

The present experiments show clearly that the masses of all the carriers studied lie in the range of tens of helium atomic masses. Thus, in all cases, models involving nearly free electrons or simple light ions seem to be excluded. The most extensive measurements, for the case of positive carriers in He II, show a mass that increases from about 40 helium masses at $1.2 \mathrm{~K}$ to approximately twice that value near $2 \mathrm{~K}$. The measurements have been shown to be quite consistent with Atkins's electrostriction model of the ion, and the temperature dependence of the mass has been shown to be a consequence of viscous hydrodynamic effects in the normal fluid component. In addition, the known mobility values in the viscous flow region have been shown to be derivable from viscous hydrodynamics, by using a radius consistent both with theory, the present mass measurements, and other determinations of the size of the carrier.

In the case of the negative carrier in liquid helium II, only a limited amount of low-quality data were taken. The effective mass is found to be $120(+90,-30)$ helium masses, a value in the range expected for an electron in a bubble state. The value of the bubble radius implied by this mass is consistent with most other experimental and theoretical evaluations. An analysis of the mobility in the viscous region seems to imply a lower value for the bubble radius.

The single measurement for a positive ion in saturated helium vapor at the normal boiling point yields a mass of $75 \pm 20 \mathrm{~m}_{\mathrm{He}}$. This number and the measured mobility are consistent with the idea that a droplet of liquid helium forms on the positive ion.

\section{ACKNOWLEDGMENTS}

We are indebted to L. Meyer, J. Northby, and M. Kuchnir for allowing us to use unpublished data. Important advice and assistance has also been received from W. Weyhmann and R. L. Garwin. We are especially indebted to W. Zimmermann for pointing out to us the necessity of considering viscous effects in calculating the effective mass and for his analysis of the electrostrictive contribution to the effective mass. We are indebted to A. O. C. Nier, M. Hamermesh, and the School of Physics of the University of Minnesota for their support. 
During part of the period in which this work was preformed one of us (A.J.D.) was a National Science Foundation Cooperative Predoctoral Fellow, and the other (T.M.S.) was an Alfred P. Sloan Foundation Fellow.

\section{REFERENCES}

1. A preliminary account of this work has been given by A. J. Dahm and T. M. Sanders, Jr., Phys. Rev. Letters 17, 126 (1966).

2. For a general review of the topic see G. Careri in Progress in Low Temperature Physics, Vol. III, edited by C. J. Gorter (North-Holland Publishing Co., Amsterdam, Netherlands, 1964), pp. 58-79.

3. G. Careri, F. Scaramuzzi, and J. O. Thompson, Nuovo Cimento 13, 186 (1959).

4. L. Meyer and F. Reif, Phys. Rev. 123, 727 (1961).

5. K. W. Schwarz and R. W. Stark, Phys. Rev. Letters 21, 967 (1968); 22, 1278 (1969).

6. G. Careri, U. Fasoli, and F. S. Gaeta, Nuovo Cimento 15, 774 (1960).

7. K. R. Atkins, Phys. Rev. 116, 1339 (1959).

8. R. A. Ferrell, Phys. Rev. 108, 167 (1957).

9. C. G. Kuper, Phys. Rev. 122, 1007 (1961).

10. B. E. Springett, Morrell H. Cohen, and Joshua Jortner, Phys. Rev. 159, 183 (1967).

11. L. Meyer, H. T. Davis, S. A. Rice, and R. J. Donnelly, Phys. Rev. 126, 1927 (1962).

12. J. L. Levine and T. M. Sanders, Jr., Phys. Rev. 154, 138 (1967).

13. P. E. Parks and R. J. Donnelly, Phys. Rev. Letters 16, 45 (1966).

14. A. V. Phelps, O. T. Fundingsland, and S. C. Brown, Phys. Rev. 84, 559 (1951).

15. T. S. Benedict and W. Shockley, Phys. Rev. 89, 1152 (1953).

16. A more detailed description of the spectrometer and other experimental apparatus is given in A. J. Dahm, Ph.D. thesis, University of Minnesota, Minneapolis, Minnesota, 1965 (unpublished).

17. G. Careri, F. Scaramuzzi, and J. O. Thomson, Nuovo Cimento 18, 957 (1960).

18. J. Herian, J. Levine, and T. M. Sanders, Jr., unpublished data.

19. G. G. Stokes, Mathematical and Physical Papers (Cambridge University Press, London, 1922), Vol. 3, p. 34; L. D. Landau and E. M. Lifshitz, Fluid Mechanics (Addison-Wesley Publishing Co., Inc., Reading, Mass., 1959), p. 96.

20. W. Rybczynski, Acad. Sci. Cracovie, Bull. 1a, 40 (1911); L. D. Landau and E. M. Lifshitz, op. cit., p. 69.

21. Mobility data were taken from F. Reif and L. Meyer, Phys. Rev. 119, 1166 (1960), L. Meyer, H. T. Davis, S. A. Rice, and R. J. Donnelly, op. cit., A. Dahm, J. Levine, J. Penley, and T. M. Sanders, Jr., Proceedings of the Seventh International Conference on Low-Temperature Physics, Toronto, 1960 , edited by G. M. Graham and A. C. Hollis Hallet (University of Toronto Press, Toronto, Canada, 1961), p. 495, and M. Kuchnir, Ph.D. thesis, University of Illinois, Urbana, Ill. (1966). Additional data in the He II region were supplied to us by J. A. Northby.

22. J. T. Tough, W. D. McCormick, and J. G. Dash, Phys. Rev. 132, 2373 (1963).

23. R. D. Taylor and J. G. Dash, Phys. Rev. 126, 1927 (1962).

24. D. G. Henshaw and A. D. B. Woods, Phys. Rev. 121, 1266 (1961).

25. W. Zimmermann, Jr., unpublished calculations (private communication).

26. E. P. Gross, Quantum Fluids, edited by D. F. Brewer (John Wiley, New York, 1966), p. 275.

27. C. Zipfel and T. M. Sanders, Jr., Proceedings of the Eleventh International Conference on LowTemperature Physics, St. Andrews, Scotland, 1968, edited by J. F. Allen, D. M. Finlayson, and D. M. McCall (St. Andrews University, St. Andrews, Scotland, 1969), Vol. 1, p. 296.

28. James Levine and T. M. Sanders, Jr., Phys. Rev. Letters 8, 159 (1962). 